TH2D-7

\title{
Partially Prizm-Gridded FDTD Analysis for Layered Structures of Transversely Curved Boundary
}

\author{
Chieh-Tsao Hwang and Ruey-Beei Wu \\ Department of Electrical Engineering, Rm. 340 \\ National Taiwan University, Taipei, Taiwan 10617, ROC
}

\begin{abstract}
A partially prism-gridded FDTD analysis is presented to deal with layered structures with curved boundary in transverse directions. It is applied to calculate the scattering parameters of vias in multilayer packaging. The good agreement of the results with those by other methods verifies the accuracy of this analysis.
\end{abstract}

\section{Introduction}

The finite-difference time-domain (FDTD) method has found a lot of applications in dealing with various electromagnetic problems. Originally developed for structures which can fit well into Cartesian coordinates [1], it suffers from significant degradation in accuracy for structures with curved boundary due to the staircasing approximation. Several attempts have been tried to alleviate the drawback by exploiting conformity to the solution region either globally or locally. Among them a very versatile and accurate one is a recently proposed hybrid method which employs the conventional FDTD method for most of the regular region and introduces the tetrahedral edgebased finite element scheme to model the re-

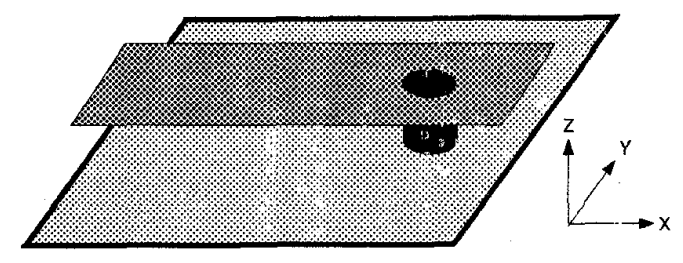

Fig.1 Geometry of microstrip with via hole ground $\left(\epsilon_{r}=2.32\right.$ and dielectric height $\left.=0.8 \mathrm{~mm}\right)$

gion near the curved surfaces [2],[3]. Numerical results of simulation validate that the hybrid method has the advantages of accuracy, flexibility, and computational efficiency.

For most microwave devices, such as planar circuits, waveguides with step transitions, and packaging interconnections, the structures can be longitudinally divided into several building layers inside which the material and conductors can be of arbitrary shape in the transverse directions. The mesh need only call for triangular cells near the curved boundary in $\mathrm{x}-\mathrm{y}$ directions while remaining rectangular grid in the z-direction. Hence, the generation of tetrahedral cells and the relatively time-consuming matrix solution of three-dimensional ninite element method can be greatly simplified. A novel hybrid method is thus proposed in this paper which employs two-dimensional hybrid FD-FEM-TD method in each layer [2] and finite difference method to link the relationship of fields between layers 


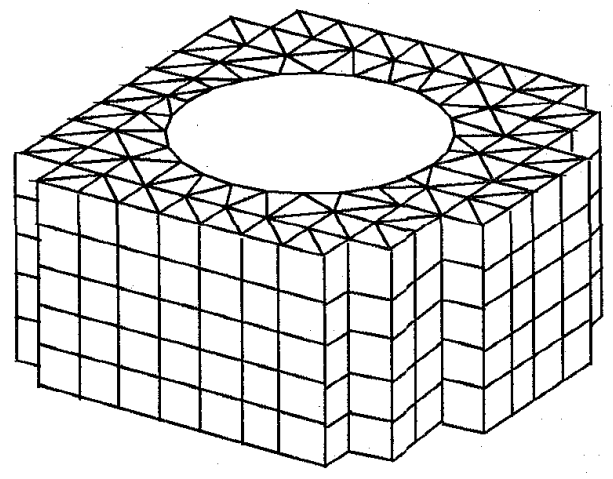

Fig.2 The prism cells around via hole

along the $z$ direction.

\section{Weak-form formulations in FEM region}

Fig.1 shows an illustrative example of microstrip line with cylindrical grounded via. Using a uniform grid in the $z$-direction, the mesh division in the $\mathrm{x}, \mathrm{y}$ plane consists of triangular elements near the via and regular FDTD grid elsewhere as shown in Fig.2. Con'sequently, there are prism cells formed by the triangular elements in the transverse plane together with the uniform grid in the $\mathrm{z}$ direction. Fig. 3 shows the field discretization in a prism cell where the transverse components $\vec{E}_{\rho}$ are located in integer $z$ coordinate layer while the longitudinal components $E_{z}$ are situated in half integer $z$ coordinate layer.

Starting from the source-free Maxwell's two curl equations in a linear isotropic region, the vector wave equation can be obtained as

$$
\nabla \times\left(\frac{1}{\mu} \nabla \times \vec{E}\right)+\epsilon \frac{\partial^{2} \vec{E}}{\partial t^{2}}=0
$$

Based on the variational reaction theory [4], (1) can be cast into the weak form by considering the inner product between (1) and an ar-

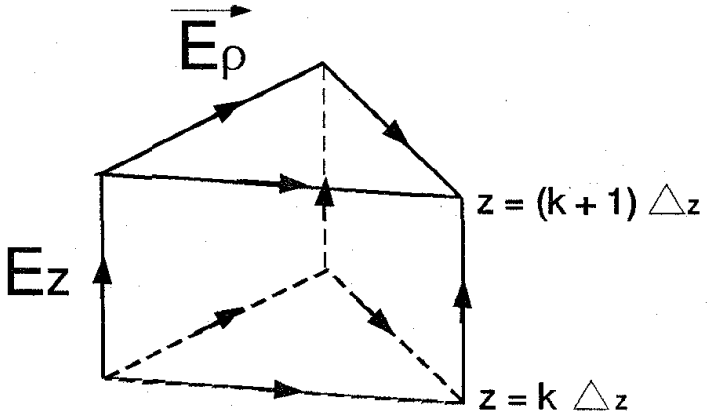

Fig.3 The prism element

bitrary testing field $\vec{E}^{a}$. Choosing transversely polarized field $\vec{E}_{\rho}^{a}$ in the $z=k \triangle_{z}$ plane as the testing field and performing some simplifications, one may obtain

$$
\begin{aligned}
& \int_{A}\left\{\frac{1}{\mu} \nabla_{\rho} \times \vec{E}_{\rho}^{a} \cdot \nabla_{\rho} \times \vec{E}_{\rho}+\epsilon \vec{E}_{\rho}^{a} \cdot \frac{\partial^{2} \vec{E}_{\rho}}{\partial t^{2}}\right. \\
& \left.+\vec{E}_{\rho}^{a} \cdot\left[\frac{\partial}{\partial z}\left(\frac{1}{\mu} \nabla_{\rho} E_{z}\right)-\frac{\partial}{\partial z}\left(\frac{1}{\mu} \frac{\partial \vec{E}_{\rho}}{\partial z}\right)\right]\right\} d S \\
& =0
\end{aligned}
$$

Here, $\nabla_{\rho}$ denotes the transverse del operator, $\vec{E}_{\rho}$ the transverse components of the electric field and $E_{z}$ the z-component. Similarly, in the $z=\left(k+\frac{1}{2}\right) \triangle_{z}$ plane, one obtain

$$
\begin{aligned}
& \int_{A} \frac{1}{\mu} \nabla_{\rho} E_{z}^{a} \cdot \nabla_{\rho} E_{z}+\epsilon E_{z}^{a} \cdot \frac{\partial^{2} E_{z}}{\partial t^{2}} \\
& -\frac{1}{\mu} \nabla_{\rho} E_{z}^{a} \cdot \frac{\partial \vec{E}_{\rho}}{\partial z} d S=0
\end{aligned}
$$

for arbitrary testing field $\vec{E}_{z}^{a}$.

The weak form (2) can be discretized into a matrix form by choosing suitable basis functions. Here, the cross-sectional area $A$ is subdivided into small triangular elements. The transverse components $\vec{E}_{\rho}$ is expanded by edge-based Whitney function, i.e.,

$$
\left.\vec{E}_{\rho}\right|_{z=k \Delta_{z}}=\sum_{i} \vec{W}_{i} e_{\rho i}=\{\vec{W}\}^{T}\left\{e_{\rho}\right\}_{k}
$$


while the longitudinal components $E_{z}$ by node-based interpolation function, i.e.,

$$
\left.E_{z}\right|_{z=\left(k+\frac{1}{2}\right) \triangle_{z}}=\sum_{i} \lambda_{i} e_{z i}=\{\lambda\}^{T}\left\{e_{z}\right\}_{k+\frac{1}{2}}
$$

The testing fields $\vec{E}_{\rho}^{a}$ and $E_{z}^{a}$ are expanded by the two corresponding basis functions. Taking integration with respect to the basis functions and applying the Ritz procedure, (2) can be reduced to two systems of coupled differential equations for transverse and longitudinal components.

Based on the Crank-Nicolson scheme, we apply central finite difference method with respect to $z$ and $t$ variables and in addition take timing average for the first term in (2) [3]. The final time marching equation in the FEM region is

$$
\begin{aligned}
{\left[G_{\rho \rho}^{+}\right]\left\{e_{\rho}\right\}_{k}^{n+1}=} & 2\left[G_{\rho \rho}^{-}\right]\left\{e_{\rho}\right\}_{k}^{n}-\left[G_{\rho \rho}^{+}\right]\left\{e_{\rho}\right\}_{k}^{n-1} \\
& -\left[C_{\rho z}\right]\left(\left\{e_{z}\right\}_{k+\frac{1}{2}}^{n}-\left\{e_{z}\right\}_{k-\frac{1}{2}}^{n}\right) \\
& +\left[D_{\rho \rho}\right]\left(\left\{e_{\rho}\right\}_{k+1}^{n}-2\left\{e_{\rho}\right\}_{k}^{n}\right. \\
& \left.+\left\{e_{\rho}\right\}_{k-1}^{n}\right)
\end{aligned}
$$

and

$$
\begin{aligned}
{\left[G_{z z}^{+}\right]\left\{e_{z}\right\}_{k+\frac{1}{2}}^{n+1}=} & 2\left[G_{z z}^{-}\right]\left\{e_{z}\right\}_{k+\frac{1}{2}}^{n}-\left[G_{z z}^{+}\right]\left\{e_{z}\right\}_{k+\frac{1}{2}}^{n-1} \\
& +\left[C_{\rho z}\right]^{T}\left(\left\{e_{\rho}\right\}_{k+1}^{n}-\left\{e_{\rho}\right\}_{k}^{n}\right)(5 b)
\end{aligned}
$$

in which

$$
\begin{aligned}
& {\left[G_{\rho \rho}^{ \pm}\right]=\int_{A} \epsilon_{r}\{\vec{W}\}\{\vec{W}\}^{T} d S} \\
& \pm \frac{\kappa^{2}}{4} \int_{A} \frac{1}{\mu_{r}}\left\{\nabla_{\rho} \times \vec{W}\right\}\left\{\nabla_{\rho} \times \vec{W}\right\}^{T} d S \\
& {\left[C_{\rho z}\right]=\kappa^{2} \int_{A} \frac{1}{\mu_{r}}\{\vec{W}\}\left\{\nabla_{\rho} \lambda\right\}^{T} d S} \\
& {\left[D_{\rho \rho}\right]=\kappa^{2} \int_{A} \frac{1}{\mu_{r}}\{\vec{W}\}\{\vec{W}\}^{T} d S} \\
& { }_{,}\left[G_{z z}^{ \pm}\right]=\int_{A} \epsilon_{r}\{\lambda\}\{\lambda\}^{T} d S \\
& \pm \frac{\kappa^{2}}{4} \int_{A} \frac{1}{\mu_{r}}\left\{\nabla_{\rho} \lambda\right\}\left\{\nabla_{\rho} \lambda\right\}^{T} d S
\end{aligned}
$$

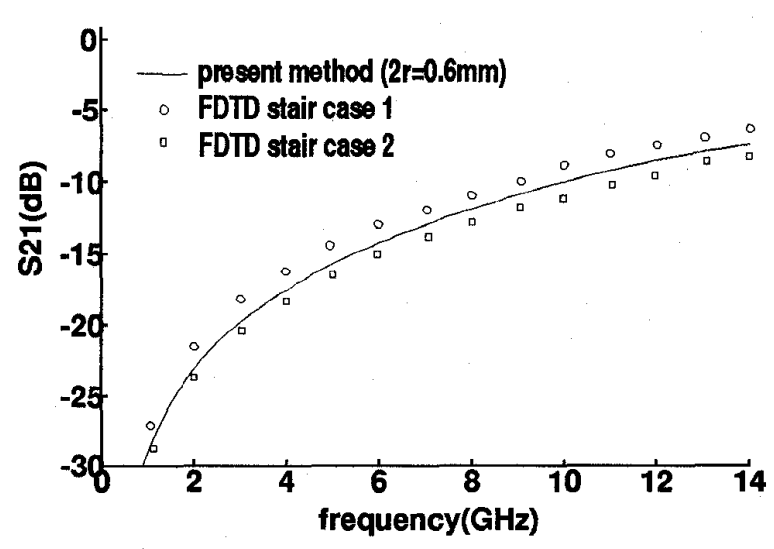

Fig.4 Comparison of the calculated $|S 21|$ with staircasing FDTD method

Here, $\kappa=\frac{\Delta_{t}}{\sqrt{\mu_{0} \epsilon_{0}} \Delta}$ is stability factor, the superscript of $\left\{e_{\rho}\right\}$ and $\left\{e_{z}\right\}$ stands for the time step and the subscript denotes the step in $z$ coordinate. The time marching scheme is the same as that described in [3], except that the electric field of unregular regions is computed by (5) layer by layer at each time step.

\section{Numerical results}

\section{A. Grounded via}

This hybrid method has been employed to characterize the microstrip with cylindrical grounded via shown in Fig.1. Fig.4 compares the calculated scattering parameters with those by two staircasing FDTD analyses [5], for which model 1 approximates the via using the inner bounded staircase while model 2 the outer bounded staircase. As expected, the results by the present hybrid method lie somewhere in between.

Fig. 5 compares the calculated results with those by mode matching method [6]. Good agreement can be noticed. 


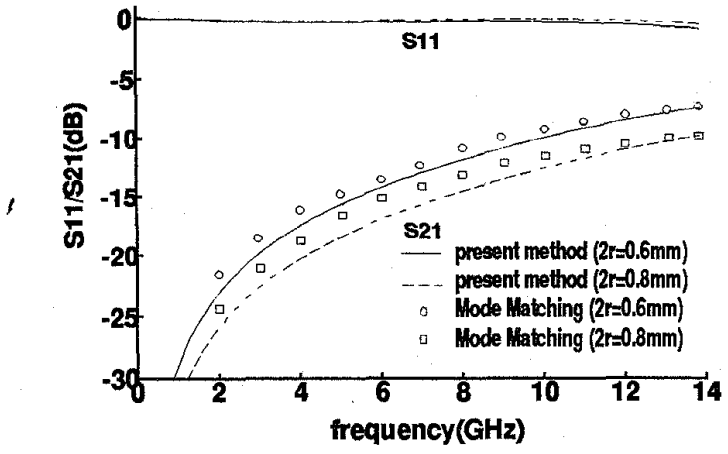

Fig. 5 Comparison of the calculated $|S 21|$ with mode matching method

\section{B. Through hole via}

The approach is also applied to deal with the through hole vias in multilayer packaging structures as shown in the inset of Fig.6. The region near the rod is modeled by prismgridded FEM. Fig. 6 compares the calculated results with those by measured [7]. Good agreement verifies that the present method is capable of characterizing objects with curve boundary.

\section{References}

, [1] K. S. Yee, "Numerical solution of initial boundary value problems in isotropic media," IEEE Trans. Antennas Propagat., vol. 14, pp.302-307, May 1966.

[2] R. B. Wu and T. Itoh, "Hybridizing FDTD analysis with unconditionally stable FEM for objects of curved boundary," IEEE MTTS 1095 Int. Microwave Symp. Dig., pp.833-836, May 1995.

[3] R. B. Wu and T. Itoh, "Hybrid finite-difference time-domain modeling of curved surfaces using tetrahedral edge el-

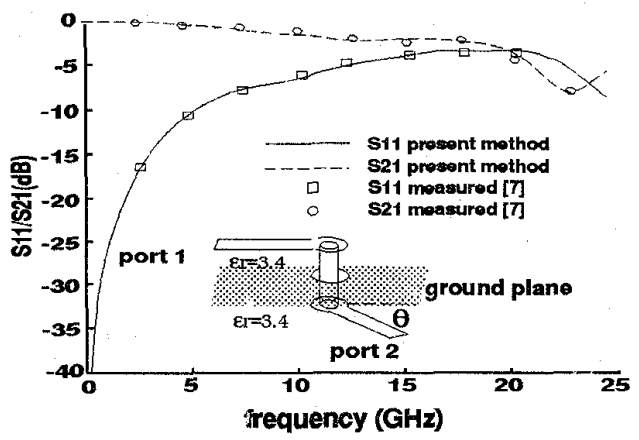

Fig.6 Comparison of the calculated $|S 11|$ and $|S 21|$ with measured results [7] (Each width of microstrips is $3.3 \mathrm{~mm}$ and the diameter of the rod and the clearance hole is $1.5 \mathrm{~mm}$ and $3.9 \mathrm{~mm}$ respectively. $\theta=0^{\circ}$ )

ements," IEEE Trans. Antennas Propagat., vol. 45, pp.1302-1309, Aug. 1997.

[4] R. B. Wu, "A wideband waveguide transition design with modified dielectric transformer using edge-based tetrahedral finite element analysis," IEEE Trans. Microwave Theory Tech., vol. 44, pp.10241031, July, 1996.

[5] D. Koh, H. B. Lee, and T. Itoh, "A hybrid full-wave analysis of via hole grounds using finite difference and finite element time domain methods," IEEE MTT-S Digest, pp.89-92, June, 1997.

[6] R. Sorrentino, F. Alessandri, M. Mongiardo, G. Avitabile, and L. Roselli, "Full-wave modeling of via hole grounds in microstrip by three-dimensional mode matching technique,"IEEE Trans. Microwave Theory Tech., vol. 40, pp.22282234, Dec. 1992.

[7] S. Maeda, T. Kashiwa, and I. Fukai, "Full wave analysis of propagation characteristics of a through hole using the finitedifference time-domain method," IEEE Trans. Microwave Theory Tech., vol. 39, pp.2154-2159, Dec. 1991. 